\title{
Elementos clave para la gestión de la creatividad
}

\author{
Rosa María Fuchs Ángeles \\ International M.B.A. por el Instituto de Empresa Business School, Madrid-España. \\ Profesora e Investigadora del Departamento Académico de Administración de la \\ Universidad del Pacífico, Lima, Perú. \\ Federico Carlo Franceso Tonetti \\ International M.B.A. por el Instituto de Empresa Business School, Madrid-España. \\ Estudios de Business Administration en la Universita' Commerciale \\ L.Bocconi en Milán. \\ Strategy Manager Europe en Lafarge-París, líder mundial en la \\ industria de cemento y hormigón.
}

\begin{abstract}
Resumen
Las organizaciones se encuentran en la búsqueda permanente de la innovación, y para alcanzarla deben fomentar la creatividad. El presente artículo analiza una serie de elementos que se tienen en cuenta en la gestión de la creatividad organizacional y propone un modelo con los factores indispensables que las organizaciones deberían considerar para lograrla.

Los elementos que se analizan son: la cultura y el entorno organizacional, la estrategia, la estructura, la comunicación, el contacto con el cliente, los mecanismos de recursos humanos (como el reclutamiento, el entrenamiento, el diseño de puestos, la compensación, la promoción y evaluación del desempeño), la orientación en el largo plazo y, finalmente, el ciclo de vida organizacional. A partir del análisis de estos elementos, se identificaron los pilares que se consideran indispensables para la gestión de la creatividad. El modelo propuesto está conformado por 5 pilares: la alineación entre estrategia, cultura y estructura organizacionales, llamada por los autores «Santísima Trinidad», la publicidad interna, la voz del consumidor, el reconocimiento y la mirada al futuro. Posteriormente, se presenta el caso de una empresa innovadora peruana desde la perspectiva del modelo y las conclusiones del estudio.
\end{abstract}

\section{Palabras clave}

Creatividad, Innovación, Cultura organizacional, Ciclo de vida organizacional

\section{Introducción}

En un entorno permanentemente competitivo, las organizaciones se encuentran en la búsqueda permanente de la innovación, y para alcanzarla deben fomentar la creatividad. En este ar- tículo se analizan los principales elementos que se deben tener en cuenta en la gestión de la creatividad organizacional. Asimismo, se propone un modelo con los factores que las empresas deben trabajar alineadamente con el fin de lograr innovaciones. 
Existen diferentes modelos para clasificar las innovaciones, según el efecto que causan en el conocimiento tecnológico y en el mercado del fabricante (Abernathy y Clarck 1985), según el régimen de apropiabilidad (Teece 1986) entre otros. Se entenderá por innovación, para efectos de este artículo, el momento en el que una nueva idea es llevada al mercado y genera un cambio positivo o añade valor a los clientes, lo que finalmente lleva a mejoras de las condiciones de vida. La clasificación que se propone es la de innovación incremental e innovación radical.

\section{Innovación incremental}

Se definirá esta innovación como evolutiva más que revolucionaria. En el anexo 1 se muestran las principales características de los cambios incrementales y los cambios radicales.

En este caso, la innovación será fruto de combinar creativamente proyectos, productos o servicios previos. Se podría considerar como el tipo de innovación más usado por las compañías, debido simplemente a que no es tan común lograr inventos revolucionarios. Este tipo de innovación ofrecerá tanto un nivel de diferenciación medio alto como ventajas clave, como menor inversión en Investigación y Desarrollo (I\&D), porque ya existen experiencias pasadas en la propia organización y mejor conocimiento de los distribuidores y los clientes. Todo esto se puede traducir en un menor riesgo. Apple nos ofreció un buen ejemplo de innovación evolutiva cuando introdujo el Ipod y el programa Itunes. En el momento del Ianzamiento, Apple no era el primero en incursionar en dispositivos digitales musicales. Sin embargo, logró combinar el uso amigable, una buena presentación y un precio adecuado.

\section{Innovación radical}

Este tipo de innovación le ofrece a las compañías la oportunidad de dar un salto y dejar a sus competidores atrás. Esta oportunidad se podría transformar en una ventaja competitiva en el largo plazo, si la innovación tiene llegada al mercado. 3M ofrece ejemplos de este tipo con su cinta Scotch y las notas Post-it. Una consecuencia de este tipo de innovación es la posibilidad de patentar la nueva tecnología o producto, lo cual representa el mejor escenario para las compañías, pues pueden esperar la maximización de sus flujos de caja y ganancias. Por otro lado, este tipo de innovación no se da con frecuencia y representa un alto riesgo para las compañías por los recursos necesarios para conseguirla.

\section{Creatividad e innovación}

Sea cual fuere el tipo de innovación que se desarrolle, tendrá detrás un cierto nivel de creatividad. Esto quiere decir que, por lo menos, una persona fue capaz de ver algo (un problema, un producto, una carencia) desde un punto de vista distinto y generar una solución o mejora no descubierta con anterioridad. Se supone que mientras las compañías se mueven hacia una innovación radical, será necesario un mayor grado de creatividad. Los conceptos de creatividad e innovación se relacionan, pero no existe innovación sin un cierto grado de creatividad. La creatividad de individuos y personas es el punto de partida para la innovación, es la semilla para la innovación (Amabile y otros 1996).

La relación entre creatividad e innovación lleva a las empresas que desean ser innovadoras a gestionar adecuadamente la creatividad. Esto, que aparentemente es obvio, no siempre es fácil de conseguir. Algunos estilos de gestión o tareas que cumplir por las organizaciones pueden inhibir la creatividad.

En la primera sección se presentan los objetivos del artículo. En la segunda, el marco conceptual, en el que se encontrarán los elementos que deben ser considerados en la gestión de la creatividad. A lo largo de esta sección se presentarán ejemplos de empresas innovadoras. En la tercera, se presenta el modelo de los 5 pilares para la creatividad; y en la cuarta, el caso de la empresa peruana 'Disfruta', desde la perspectiva del modelo. La quinta sección presenta los principales resultados $y$, finalmente, la sexta sección, las conclusiones que se derivan del estudio realizado. 


\section{Objetivos}

- Explorar, mediante ejemplos de empresas innovadoras, los principales elementos que se deben gestionar para fomentar la creatividad.

- Definir un modelo con los elementos imprescindibles para la gestión de la creatividad.

- Analizar la empresa 'Disfruta' bajo la perspectiva del modelo desarrollado.

\section{Marco Conceptual}

\section{Cómo gestionar el proceso de creatividad-innova- ción en una empresa}

Se explorarán algunos elementos que se consideran importantes para lograr que las empresas puedan gestionar la creatividad.

\section{Cultura y entorno organizacional}

¿Existe una cultura organizacional que promueva la creatividad? Se tomará la propuesta de Daft (2004), elaborada sobre los trabajos realizados por Denison y Mishra (1995), Hooijberg y Petrock (1993) y Quinn (1988), que presenta los tipos de cultura corporativa (véase el anexo 2). Una cultura que promueva la creatividad requiere una estrategia con enfoque en el ambiente externo, porque las fuentes para lograr innovaciones van más allá de los recursos internos de la organización. Las áreas de marketing o de I\&D forman parte de una red que se extiende hasta los clientes, proveedores, competidores y otros grupos de interés. De manera adicional, el entorno organizacional cambia rápidamente, razón por la cual una organización necesita altos niveles de flexibilidad. Por lo tanto, una organización que fomenta la creatividad y busca la innovación debería tener una cultura adaptable.

La cultura organizacional de $3 \mathrm{M}$ ilustra este punto, sus miembros describen que en $3 \mathrm{M}$ «existe un clima que estimula que la gente ordinaria tenga resultados extraordinarios». En compañías que buscan la innovación, como 3M, Google y la empresa india Mahindra \& Mahindra, se promueve la experimentación, se contrata personas con opiniones propias y se es consciente de la importancia de correr riesgos afrontando fracasos.

\section{Estrategia}

¿Cuál será el tipo de estrategia que pueda promover la creatividad y lograr los mejores resultados? Un enfoque en diferenciación más que en costos (Porter 1982) debería estar presente. En otras palabras, un enfoque de valor tanto para la compañía como para los clientes. La estrategia también debería contar con orientación de largo plazo. Aunque se espera que la innovación genere ganancias en el mediano y largo plazo, de ningún modo las empresas pueden descuidar los productos y servicios que les rinden en el corto plazo. Seguir una estrategia de pionero o buscador (Miles y Snow 1978) exige innovaciones más rápidas, se corren riesgos pero se busca una reputación de líder y el mejor posicionamiento. La ventaja del pionero es que podrá definir las nuevas reglas de competencia.

\section{Estructura}

La estrategia y el entorno que enfrenta cada organización determinarán su estructura. ¿Cuál es la mejor estructura para la innovación? Se podría pensar que la estructura orgánica. Sin embargo, depende del enfoque que una compañía le quiera dar a la innovación. Existen compañías que consideran que la innovación se encuentra en un departamento, como I\&D, y adoptan una estructura mecánica. No obstante, en las empresas que se caracterizan por ser innovadoras, la innovación es demasiado importante para dejarla en manos de un departamento. Como De Bono sostuvo en una conferencia (2004: 13), «cuestionar el statu quo debería ser parte del trabajo de cada empleado, empezando por el gerente».

\section{Comunicación}

¿Cómo se logra que la empresa sea receptiva a una cultura de creatividad? Se necesita de un alto grado de interiorización de todos los agentes involucrados en la empresa, lo que implica una comunicación efectiva entre la alta dirección y los empleados. Para ello, es conveniente que haya políticas específicas que reflejen y comuniquen 
la intención de la empresa por abandonar aquello que obstaculiza el trabajo y que destruye valor. Estas políticas deben ser publicadas, conocidas al interior de la organización y deben ser intensas (Tushman y O'Relly 1998). Como parte de las políticas de la empresa, debe considerarse la realización de sesiones en las que se compartan experiencias de éxito innovador de alguna unidad de la empresa. En estas sesiones se aprende mucho y se generan nuevas ideas que pueden ser aplicadas en otras unidades.

Por ejemplo, Saatchi \& Saatchi, reconocida compañía publicitaria, determinó que quiere ser la «fábrica de las ideas». Saben que las ideas son su ventaja competitiva, por lo que han organizado su empresa sin divisiones. Uno de sus principios dice: «las divisiones dividen». En una visita a la oficina de Madrid, se pudo comprobar que el área de contabilidad trabaja en mesas redondas, en equipos que se comunican permanentemente, compartiendo clientes. En el área creativa, las personas trabajan en un amplio salón sin divisiones, si bien cada uno tiene su espacio trabajan juntos. La compañía fomenta que todos los miembros generen ideas y las compartan no solo en un mismo país, sino con todas las sucursales, por medio de la Intranet. Los viernes se muestran los comerciales a todos los empleados, quienes pueden criticar y dar ideas. Para motivar la creatividad cuentan con todas las facilidades posibles: salones de discusión, sofás confortables, entre otras.

Como se puede apreciar, promover la innovación requiere tiempo y congruencia absoluta por parte de la alta dirección, así como la repetición constante del mensaje por medio de buenas normas establecidas. Todos los miembros de la empresa deben compartir el mensaje, si se desea impulsar la creatividad.

\section{Contacto con el cliente}

La innovación implica salir a observar, preguntar y escuchar a los clientes y vendedores (Drucker 1997). Implica analizar cifras, conocer las expectativas, los hábitos, los comportamientos, para encontrar la oportunidad o la necesidad insatisfecha; y en función de ella, desarrollar la innovación. Si ello no se realiza, la oportunidad será aprovechada por la competencia. Si la empresa no está enfocada desde un inicio en el mercado, se dejará de lado a aquellos clientes que quedaron fuera de la vista de la empresa, quienes constituyen mercados potenciales que deben ser aprovechados. En resumen, las empresas deben entender que los productos son definidos por el mercado y no por el productor; que ninguna empresa aprovechará la oportunidad para innovar, si no invierte en tareas de campo y cuestiona constantemente el uso, la utilidad y el valor que los productos otorgan a los clientes.

Un ejemplo exitoso de acercamiento al cliente para lograr innovaciones lo tiene 3M. La introducción del sistema Lead Users fue quizá uno de sus logros más significativos. El sistema consiste en juntar equipos de 4 a 6 personas con miembros del área técnica y de marketing, que van a explorar áreas de desarrollo y luego contactarán a los líderes usuarios para trabajar con ellos.

En Siemens se convencieron de que enfocarse en el cliente es crucial. Especialmente en el área de telecomunicaciones, entender al cliente y su necesidad de integrar los diferentes dispositivos se convirtió para ellos en una necesidad. En HP, Carly Fiorina sostuvo que era tiempo de impulsar lo que los clientes desean: sistemas y soluciones, no componentes (Waters, London y Morrison 2004). Después de que Carly Fiorina se convirtió en CEO de $\mathrm{HP}$, se inició un cambio en su estructura. En el año 2000 se crearon áreas transversales a la organización, específicamente para tratar asuntos de clientes. Esto representó un shock cultural para los empleados. Fiorina buscaba un contacto más cercano con los clientes, mientras se mantenía la innovación. Ambas compañías tomaron en cuenta la necesidad de revitalizar y cambiar la estructura para trabajar junto al cliente.

\section{Mecanismos de recursos humanos}

El área de Recursos Humanos tendrá que prestar especial atención al alineamiento de la estrate- 
gia, la cultura, el entorno, y a la consistencia entre los procesos de recursos humanos. No es la intención de este artículo realizar sugerencias sobre mejores prácticas, sino mostrar lo que se hace para impulsar la innovación en empresas consideradas innovadoras.

\section{Reclutamiento}

Las habilidades requeridas para una persona innovadora, según la empresa Siemens, son: comunicación, colaboración e involucramiento. Para $3 \mathrm{M}$, la principal razón de su éxito es el recurso humano, que se describe como talentosa e innovadora y que es catalogada como personas comunes y corrientes con espíritu emprendedor. En este nivel se considera emprendedor a quien está abierto a nuevas ideas, es perseverante, tiene capacidad para enfrentarse a los superiores y es capaz de encontrar soluciones.

\section{Entrenamiento}

Cuando un trabajador nuevo llega a una empresa que desea promover la innovación, son importantes los programas de mentoring. En $3 \mathrm{M}$ consideran que el mentoring sirve para introducir la cultura, para transmitir el clima de apertura. También ayudan los programas específicos como el de Samsung, al contratar a IDEO para brindar talleres de creatividad a sus diseñadores.

\section{Diseño del puesto}

Para promover la creatividad, la autonomía y el significado de la tarea son muy importantes, como lo señala el modelo de las características del trabajo de Hackman y Oldham (1976). Cuando se brinda autonomía, la empresa está compartiendo responsabilidad con sus empleados. La autonomía significa brindar libertad para organizar el trabajo, para decidir qué hacer, pero siguiendo los intereses y normas de la organización. Además, el hecho de que el trabajador sea consciente de la relevancia de su trabajo frente a los resultados de la compañía, puede ser un gran motivador para innovar. En $3 \mathrm{M}$ tienen la regla del $15 \%$, que establece que los trabajadores pueden usar el $15 \%$ de su tiempo para pensar y trabajar en lo que ellos quieran. En este $15 \%$ surge la mayor parte de sus inventos. En Samsung se plantearon que querían ser el 'Mercedes' de los electrodomésticos, razón por la cual organizaron su área de diseño que no cuenta con un código de vestir y donde se anima a enfrentar a los superiores. Todos son tratados por igual, sin importar la jerarquía.

En Google, el presupuesto para innovación está en función del tiempo de los empleados y forma parte de las descripciones del puesto. Normalmente, los empleados son quienes generan las nuevas ideas. Los empleados técnicos son obligados a pasar el $80 \%$ de su tiempo en el negocio principal y el $20 \%$ restante en los proyectos que ellos mismos eligen. Si no tienen un buen proyecto para ese $20 \%$, saben que su evaluación se verá impactada de manera negativa. A los gerentes también se les pide que dediquen tiempo para la innovación, el $70 \%$ de su tiempo se dedica al negocio principal, el $20 \%$ a proyectos relacionados y el $10 \%$ a negocios y productos totalmente nuevos.

\section{Compensación}

Se debe compensar la innovación. En este punto, lo que se cuestiona es si el dinero es el mecanismo adecuado. A favor de una compensación económica, se sostiene que si una compañía obtiene ganancias con un nuevo producto es justo que comparta el éxito financiero con el innovador. En contra, se sostiene que no hay mejor compensación que ver una idea hecha realidad. El tipo de compensación dependerá del tipo de empresa, de su cultura organizacional y de la personalidad del individuo o equipo innovador. Si la innovación es parte de la cultura, será mejor no entregar incentivos económicos porque ser creativo será parte de las funciones del día a día. Es decir, recibe un sueldo por desempeñar dichas funciones. Los incentivos no monetarios estarán ligados al reconocimiento. Por ejemplo, se puede optar por premiar las mejores ideas en público o destacarlas en un boletín o ceremonia especiales. En 3M tienen un programa de reconocimiento Ilamado «Carlton 
Society» y el «Engineering Achievement Award of Excellence». Adicionalmente, en J\&J y 3M prometen a la persona que desarrolle con éxito cierta innovación que será el jefe de la división en la que se desarrolló.

\section{Promoción}

Cuando la innovación es parte de la cultura y la organización la quiere impulsar, es mejor ligarla con el desarrollo profesional. La innovación debe ser uno de los criterios para las promociones. El reto consiste en encontrar la mejor manera de medir los aportes manteniendo la transparencia. Podría medirse el número de ideas desarrolladas en un año, el número que se ha convertido en éxito, el número de ideas implementadas, pero siempre enviando el mensaje de que equivocarse está permitido. Si los empleados ven que se promueve la innovación pero que equivocarse condiciona un camino más lento hacia los ascensos, se inclinarán por opciones más conservadoras.

\section{Evaluación del desempeño}

La evaluación del desempeño deberá considerarse como una herramienta para determinar dónde invertir recursos y si las personas son las adecuadas para una cultura de innovación. Esta evaluación deberá alinear los objetivos estratégicos y las actividades de los individuos. Una empresa que busca la innovación debería medir los resultados y los comportamientos. En WestLB, banco alemán con presencia en más de treinta países, la innovación es importantísima para sus clientes corporativos. Esta innovación la logran mediante su personal; por ello, las habilidades de compartir y trabajar en equipo se evalúan más que los resultados.

\section{Orientación en el largo plazo}

Es indispensable para la gestión empresarial que se piense, desde ahora, en el largo plazo, en el futuro hacia el cual se dirige la empresa. El punto de partida, especialmente en el caso de los negocios existentes, es conocer la foto inicial de la empresa; luego, la empresa debe preguntarse cuánto tiempo más podrá mantenerse su pro- ducto en el mercado, cuándo declinará y a qué velocidad. La brecha entre las respuestas y los objetivos organizacionales permite determinar el grado de innovación necesaria para la consecución de los objetivos. Asimismo, supone la necesidad de elaborar un plan empresarial donde la innovación tenga objetivos claros (Drucker 1997).

Una visión clara, que comprometa emocionalmente, proporciona una base estratégica para los requisitos de las corrientes de innovación. Las visiones directas, sencillas y competitivas ofrecen una guía para que las unidades se muevan entre el dominio de la eficiencia y la innovación progresiva (Tushman y O'Relly 1998).

\section{Ciclo de vida organizacional}

\section{Ciclo de vida organizacional y creatividad}

La etapa del ciclo de vida de la organización debe influenciar de alguna manera la innovación en las empresas. Tomaremos como referencia el modelo de ciclo de vida organizacional propuesto por Daft (2004), que se presenta en el cuadro 1.

En este modelo se mencionan cuatro etapas relacionadas con el crecimiento de la empresa, que se analizarán a continuación, en función de la búsqueda de innovación.

\section{Etapa empresarial}

Esta es una típica etapa para la creatividad y la innovación. Normalmente, a cargo de los propios dueños. La organización necesita gerentes para que el emprendedor se concentre en el desarrollo de nuevos productos o servicios de acuerdo con sus fortalezas.

\section{Etapa de colectividad}

En esta etapa, la compañía crece y la gerencia le provee de direcciones, reglas y guías. La estructura organizacional tiene un nivel de formalización y control bajos. La creatividad y la innovación son aún estimuladas en estos «primeros años», y la creación de ideas se empieza a compartir entre el dueño y los empleados. 


\section{Cuadro 1}

Ciclos de vida organizacional

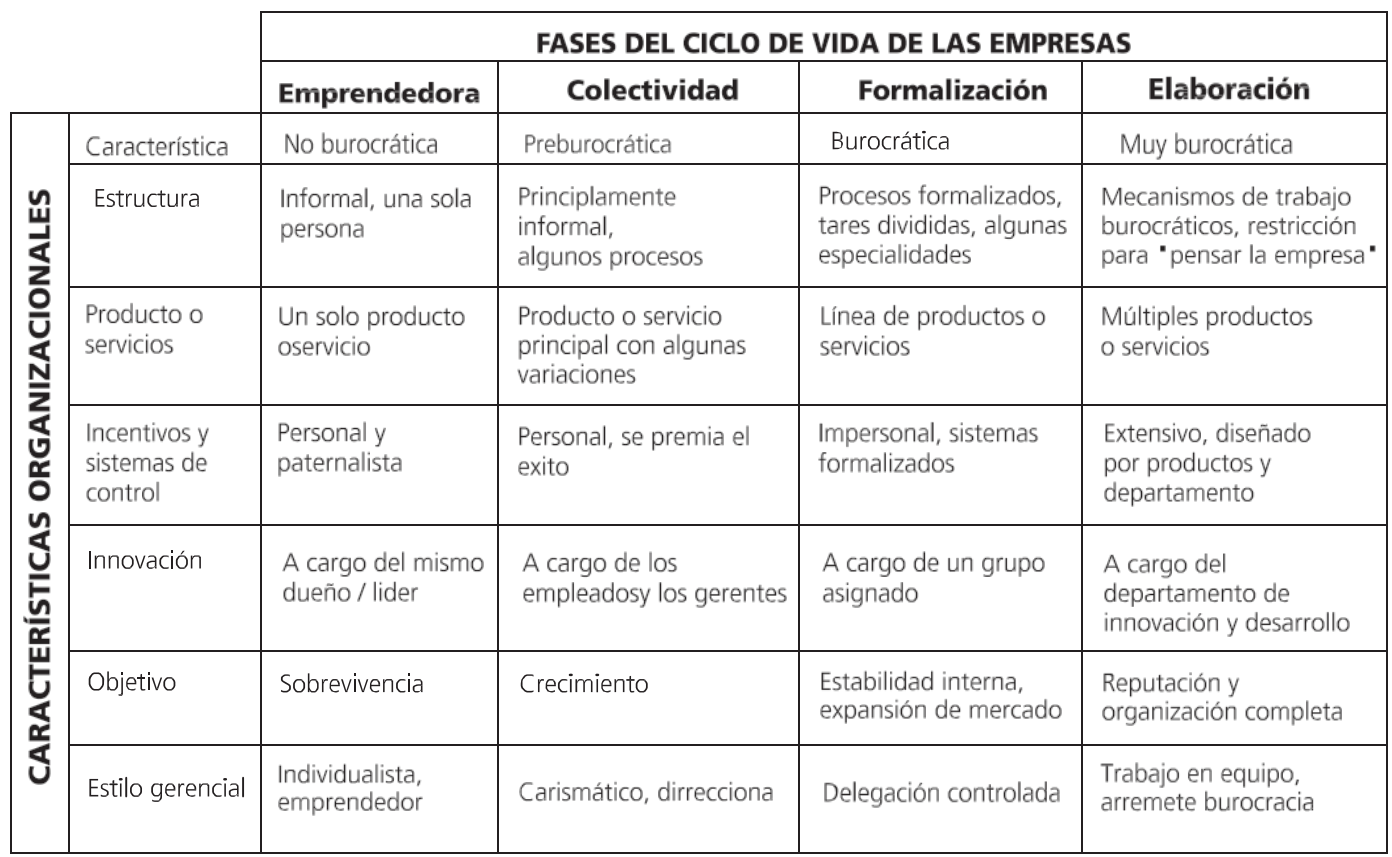

Fuente: DAFT, Richard. Organization Theory and Design. 2004. Octava Edición. Thomson South- Western

\section{Etapa de formalización}

En esta etapa, los procedimientos formales se hacen necesarios. Esto demanda que la innovación se maneje de otra manera, pasando a encargarse a grupos multifuncionales. Creemos que en esta etapa el estilo para manejar la innovación y la creatividad cambia, por encontrarse ante las presiones crecientes de los sistemas de gestión y la estructura organizacional, al punto que en los trabajadores puede aparecer algo de descontento. En este momento, un cambio cultural se hace necesario o al menos debe ser considerado. Cuanto más se incrementa el grado de control al interior de la organización, más rápido se acerca la crisis de esta etapa: la excesiva burocratización.

\section{Etapa de elaboración}

El desarrollo de trabajo en equipo y otros elementos de integración son típicos de esta etapa. Es difícil para las empresas lidiar con la creatividad y sus requerimientos. La innovación se traslada a departamentos de I\&D. Compañías como 3M, HP y Siemens trataron de encontrar un balance entre la libertad y el control, revitalizándose y adaptando su estructura para trabajar junto al cliente.

\section{Cómo innovar en empresas pequeñas}

Los ejemplos presentados en este artículo corresponden a compañías que operan globalmente y que tienen recursos para investigar y probar ideas. Por lo general, las empresas en países en desarrollo no tienen suficientes recursos y trabajan con inestabilidad política. Aun en estas circunstancias, las compañías que se encuentran en la etapa empresarial pueden alcanzar sus objetivos. Una de las estrategias que siguen es «vivir con el cliente», es decir, observar todos sus movimientos y costumbres. Por ejemplo, en el Perú, la empresa Transcontinental S.A.C. lanzó a finales de los años 1990 su producto «Arroz Costeño». En esa época, el arroz se vendía principalmente a granel y los arroces envasados se ofrecían en bolsas de un kilogramo. La compañía analizó muy 
bien al consumidor y descubrió que una familia promedio utilizaba en realidad menos de un kilogramo diariamente. Decidieron lanzar el producto en envases de tres cuartos de kilogramo, a un mejor precio, y tuvieron un éxito total, no solo en los clientes que ya ofrecían el arroz envasado sino en las personas que consumían el producto a granel. Lograron un cambio en el mercado y, actualmente, son los líderes del mercado.

\section{Modelo de los 5 pilares para la creatividad}

Cada uno de los elementos mencionados en el acápite anterior es relevante en la gestión de la creatividad. Sin embargo, luego del análisis realizado se han identificado cinco elementos que son imprescindibles, si se desea lograr el fomento de la creatividad. Como consecuencia, se propone un modelo basado en 5 pilares para gestionar la creatividad (véase el anexo 3). El cuadro 2 muestra la relación entre los elementos analizados y la propuesta de los 5 pilares para gestionar la creatividad.

\section{Cuadro 2}

Propuesta de 5 los pilares

\begin{tabular}{|l|c|}
\hline \multicolumn{1}{|c|}{ Elementos analizados } & \multirow{2}{*}{ Pilares para la creatividad } \\
\hline Cultura y entorno & \multicolumn{2}{|c|}{ Santima Trinidad } \\
\hline Estrategia & Publicidad interna \\
\hline Estructura & La voz del consumidor \\
\hline Comunicación & \\
\hline $\begin{array}{l}\text { Contacto con el cliente } \\
\text { - Rumanos }\end{array}$ & \\
\hline - Entrenamiento & Reconocimiento \\
- Diseño del Puesto & \\
\hline - Compensación & \\
\hline Orientación a largo plazo & Mirada al futuro \\
\hline \multicolumn{2}{|c|}{ Ciclo de vida organizacional: elemento transversal } \\
\hline
\end{tabular}

Fuente: Elaboración propia.
Lo que se postula es que cuanto mayor sea la consistencia entre estos cinco conceptos, más efectivo será el manejo de la creatividad e innovación en una organización. Cada uno de ellos refuerza al otro y su integración le ofrece a una organización la oportunidad de manejar el proceso de creatividad-innovación por las distintas etapas del ciclo de vida organizacional, así como de mantenerlo vivo mediante los cambios del entorno que toque enfrentar. Los pilares son:

- La Santísima Trinidad. El acoplamiento entre la cultura, la estrategia y la estructura es lo que se denomina como «Santísima Trinidad». Creemos que deben estar alineados para enfocar los recursos en lo que es relevante para la innovación. De esta manera, también se captará personas con el perfil adecuado.

- Publicidad interna. Si la innovación es un objetivo, debería estar claro y «traducirse» a todo el personal. Es decir, que todos los miembros de la organización tengan conocimiento con claridad de cómo sus funciones se relacionan con la innovación. El concepto se tiene que «publicitar internamente».

- La voz del consumidor. Las compañías deben buscar la innovación porque quieren cumplir con los requerimientos del entorno, en general, y de los consumidores en particular. Orientarse hacia el cliente determinará el éxito de los esfuerzos por innovar. Cuando existe el enfoque hacia el cliente, tanto las compañías grandes como las pequeñas, en diferentes niveles de desarrollo, pueden lograr el éxito.

- Reconocimiento. La consistencia entre todos los procesos de recursos humanos se hace necesaria, pero, particularmente, se debe prestar atención a la recompensa y a la promoción como un medio de reconocimiento para el individuo o el equipo que generó la innovación.

- Mirada al futuro. Es necesaria una orientación de largo plazo para determinar la dimensión que la innovación tendrá en la compañía. Los recursos y el soporte necesarios se determinarán en función del enfoque de largo plazo. 


\section{Caso de empresa innovadora}

Se aplicó este modelo en la empresa peruana «Disfruta», fundada en julio de 2007, que está creciendo rápidamente y con éxito. Se le pidió a la fundadora, señorita Azucena Gutiérrez, que nos explicara la evolución de la empresa y que nos detallara cuáles creía que eran los elementos fundamentales de su modelo de negocio, especialmente para fomentar la creatividad.

Disfruta nace de la idea de dos jóvenes hermanos emprendedores, Azucena y Eduardo, provenientes de una familia pujante que también había salido adelante con negocios propios. En 2007, con una inversión de 35.000 dólares, abrió su primer local en el distrito de San Borja, en Lima. A los dos meses de la inauguración, ya había superado sus proyecciones iniciales de ventas en $100 \%$. Al inicio trabajaban solo 4 personas, incluidos los dueños, $y$, en menos de dos años, dicha cifra se ha incrementado a 42, como consecuencia de una planificación adecuada, energía y creatividad, Disfruta se propuso terminar el primer año con tres locales y así sucedió. En la actualidad, planean terminar el año 2008 con 2 locales más.

Disfruta desarrolló un concepto distinto al de la juguería tradicional. Posicionaron dos medidas de vasos, terminando con la costumbre de los clientes de pedir una «yapita»; es decir, un poco más. Asimismo, posicionaron nombres creativos para los jugos, como Sinfonía Cool y Zúmbate. El mismo local tiene un concepto distinto, las mesas tienen formas de frutas y hasta en el piso se pueden apreciar algunos plátanos o manzanas. Lo fundamental, los jugos son el resultado de realizar combinaciones creativas de distintas frutas (cuentan con quince combinaciones de frutas). También han lanzado productos nuevos, como los «chocofrutas», que son bebidas calientes con frutas.

\section{Aplicación del modelo}

- La Santísima Trinidad. La cultura organizacional en Disfruta se resume en la palabra «energía», la cual tiene que estar presente en todo lo que se haga en el trabajo, ya sea en la atención, en la producción, etcétera. Se comparte una cercanía entre los dueños y el personal. Disfruta está presto a probar nuevos sabores o combinaciones, y muchas veces estos nacen de los propios productores de fruta que, desde la selva, se comunican con la empresa y le envían muestras de sus productos. La estrategia que siguen es la diferenciación, se evidencia en sus presentaciones y en la infraestructura. La estructura es empresarial (Mintzberg 1989), en donde el ápice estratégico lo constituyen tres personas y el núcleo de operaciones, el resto.

- Publicidad interna. El espíritu de energía se transmite por medio de su mantra. Los dueños llaman así al estribillo que todo el debe personal repetir y tener presente: «Disfruta, felicidad, energía y salud». En cada local comparten una pizarra que muestra hechos importantes para Disfruta, desde los recibos de servicios, para ser conscientes de los gastos, hasta frases motivadoras. En un inicio, el contenido de la pizarra estaba en manos de los dueños, pero ahora está en manos de los propios trabajadores.

- La voz del consumidor. Disfruta está permanentemente al tanto de lo que sus clientes demandan. La comunicación es directa en sus locales, por medio del personal que los atiende. El personal conoce muy de cerca a sus clientes, sus nombres y gustos. Se está implementando el tema del buzón de sugerencias y la realización de grupos de enfoque. Los dueños atienden directamente por lo menos dos veces a la semana; de esa manera, mantienen el contacto con sus clientes.

- Reconocimiento. El personal en Disfruta está constituido, en su mayoría, por jóvenes estudiantes. Cuando se realiza la selección de personal, se busca que los futuros trabajadores tengan energía, vocación de servicio y propongan soluciones (para ello se analizan sus actividades extracurriculares). El proceso de inducción está en manos de los propios dueños y en él dan a conocer su mantra. Las capacitaciones relacionadas con los temas de servicio al 
cliente se realizan cada tres meses. Se otorga un reconocimiento monetario por ventas y cuando se trata de ideas creativas, el reconocimiento es público. Las oportunidades de crecimiento están en función de la experiencia de negocio que vaya generando cada trabajador.

- Mirada al futuro. Si Disfruta es tan exitosa, en parte, se debe a que desde que era un proyecto creció de manera ordenada, gracias a las metas claras que tenían sus fundadores. Tal vez, en el día a día, fueron aprendiendo cuáles eran las estrategias que debían seguir o los errores que no se debían repetir. Sin embargo, lo que siempre tuvieron claro es que querían trabajar con frutas, desarrollar el concepto de juguerías y apostar por el crecimiento en ca- dena. Disfruta se proyecta con más locales en el Perú y el extranjero, con una planta que trabaja con más variedades de frutas y con una integración hacia adelante y hacia atrás.

\section{Resultados}

Se reconoce a Disfruta como una empresa innovadora y en la etapa del ciclo de vida de colectividad. El alineamiento de los elementos que se analizan en el modelo propuesto es el que logra que Disfruta sea una empresa con espíritu emprendedor, que fomenta la creatividad y que ha logrado posicionar un concepto innovador en un sector tradicional. En el cuadro 3 se presenta un resumen de los aspectos analizados. 
Cuadro 3

Disfruta: resultados de la aplicación del Modelo de los 5 pilares para la creatividad

\begin{tabular}{|c|c|}
\hline Pilares & Resultado \\
\hline La Santísima Trinidad & $\begin{array}{l}\text { La cultura, la estrategia y la estructura en Disfruta se encuentran alineadas. Existe } \\
\text { consistencia entre las tres. } \\
\text { La cultura es adaptable, la estrategia es la de diferenciación y la estructura es del } \\
\text { tipo empresarial. } \\
\text { Se puede resumir su cultura en la palabra «energía». La energía está presente en } \\
\text { sus gerentes y en todo el personal del área de producción y de atención al público. } \\
\text { La cercanía y confianza entre los dueños y los trabajadores también es una caracte- } \\
\text { rística importante. }\end{array}$ \\
\hline Publicidad interna & $\begin{array}{l}\text { Todos los miembros de la organización reconocen que ser creativos es parte de sus } \\
\text { funciones. Los fundadores lo transmiten de diversas maneras. } \\
\text { El mantra de la empresa es: «Disfruta, felicidad, energía y salud». } \\
\text { Uno de los elementos más importantes de la comunicación interna es una pizarra } \\
\text { con la que cuentan todos los locales. El hecho de reflejar en ella los temas de } \\
\text { interés, se ha interiorizado en los propios trabajadores, quienes ahora son los que } \\
\text { tienen a su cargo el contenido de las pizarras. }\end{array}$ \\
\hline La voz del consumidor & $\begin{array}{l}\text { La orientación hacia el cliente guía las labores de Disfruta. La capacitación de los } \\
\text { trabajadores que ocupan los puestos de atención al cliente se realiza de tal manera } \\
\text { que generen una relación cercana con el cliente. Llegan a conocer los nombres y } \\
\text { los gustos de los clientes. Un elemento importante es que los propios dueños, a } \\
\text { pesar de sus recargadas agendas, se turnan en los puestos de atención al público. } \\
\text { Gracias a ello, no dejan de recibir retroalimentación directa por parte de sus clien- } \\
\text { tes, quienes les hacen ver las fortalezas y los aspectos por mejorar. }\end{array}$ \\
\hline Reconocimiento & $\begin{array}{l}\text { El esfuerzo innovador y de alerta al mercado es permanentemente reconocido. } \\
\text { Disfruta prefiere, para estos casos, el reconocimiento público y no el monetario. } \\
\text { Los trabajadores que aportan ideas innovadoras o que detectan problemas por solu- } \\
\text { cionar son tomados como ejemplos a seguir. } \\
\text { El tema de la línea de carrera también se toma en cuenta en Disfruta. }\end{array}$ \\
\hline Mirada al futuro & $\begin{array}{l}\text { La orientación de largo plazo ha logrado que, desde el principio, cada uno de los } \\
\text { retos de Disfruta hayan sido bien formulados y se hayan realizado. } \\
\text { Los propietarios están siempre proyectándose a futuro, trabajando con contactos y } \\
\text { redes para poder alcanzar las metas que se trazan. } \\
\text { Si bien la experiencia la van ganando día a día, lo que los guía es la claridad en los } \\
\text { objetivos que se trazan para el largo plazo. }\end{array}$ \\
\hline
\end{tabular}

Fuente: Elaboración propia. 


\section{Conclusiones}

- Manejar la cultura organizacional de acuerdo con la etapa del ciclo de vida en la que se encuentra la empresa es clave. Moverse de una etapa a otra puede afectar a una compañía en sus objetivos, estrategia, estructura, entre otros aspectos. Por ello, su cultura debe ser consistente con estos cambios y, si fuese necesario, ir adaptándose a ellos. Tener una cultura del tipo adaptable favorece la creatividad.

- Los líderes desempeñan un papel fundamental desde la etapa de colectividad. Ellos son los que deben percibir la necesidad de cambio, anticipar la próxima crisis organizacional, planear las medidas para contrarrestarlas y asegurar que la información se comparta en la empresa.
- La importancia del trabajo en equipo y las coordinaciones entre áreas crecen mientras que la organización lo hace. Cuanto mayor es la formalización, mayor es la necesidad de integración horizontal y de compartir información para mantener la innovación y la creatividad vivas.

- Los factores discutidos en este artículo deben ser considerados por las empresas que desean contar con la creatividad e innovación como eje central de su modelo de negocios.

- El alineamiento de los cinco elementos mencionados en el modelo constituye la base para que en las empresas surja la creatividad y, de esta manera, se fomente la innovación. 


\section{Referencias}

3M Company

2002 A century of innovation. The $3 \mathrm{M}$ story. $1^{\mathrm{a}}$ edición. Estados Unidos: 3M Company.

ABERNATHY, William y CLARCK, Kim

1985 «Innovation: Mapping the winds of creative destruction», Research Policy 14 (United States), pp. 3-22.

AFUAH, Allan

1999 «Modelos de innovación». En: La dinámica de la innovación organizacional». México: Oxford University Press, pp. 17-57.

AMABILE, Teresa; Regina CONTI, Heather COON, Jefrey LAZENBY y Michael HERRON

1996 «Assessing the work environment for creativity». En: The Academy of Management Journal, Vol. 39, № 5, octubre, pp. 11541184.

\section{BARLETT, Christopher y Afroze MOHAMED}

1995 «3M profile of an Innovating Company». Harvard Business School Case, No 9-395016. Cambridge, MA: Harvard Business Publishing, 3 de enero.

\section{BETTENCOURT, Lance y Anthony ULWICK}

2008 «The Customer-Centered Innovation Map». En: Harvard Business Review, Vol. 86, № 5. Cambridge, MA: Harvard Business Publishing, mayo, pp. 109-114.

BIRKINSHAW, Julian; Rick DELBRIDGE y John BESSANT

2004 «A leap into the unknown». En: Financial Times, 17 de setiembre, pp. 10-11. Special Report «Mastering Innovation», primera parte.

\section{CAMPBELL, Andrew}

2004 «The dangers of being distracted». En: Financial Times, 17 de setiembre, pp. 4-5. Special Report «Mastering Innovation», primera parte.pp. 4-5.
CLEMONS, Eric y Rick SPITLER

2004 «The new language of consumer behavior». En: Financial Times, 1 de octubre, pp. 4-5. Special Report «Mastering Innovation», tercera parte.

DAFT, Richard

2004 Organization Theory and Design. 8va. edición. Thomson South-Western College.

DE BONO, Edward

2004 «Open your mind to change with new opportunities». Conferencia Anual de Antiguos Alumnos del Instituto de Empresa. España, Madrid, Instituto de Empresa Business School, pp. 13-16.

DENISON, Daniel y Aneil MISHRA

1995 «Toward a Theory of Organizational Culture and Effectiveness». En: Organization Science 6, No, 2, Marzo- Abril, pp.204-223.

DIMOCK, Marshall y Bethel VERMONT

1986 «Creativity». En: Public Administration Review, Vol. 46, № 1, enero-febrero, pp. 3-7.

DRUCKER, Peter

1997 «El empresario innovador», «Innovación con objetivos», «ldea brillante», «Principios de la innovación». En: La innovación y el empresario emprendedor: la práctica y los principios. Barcelona: Apóstrofe, pp. 43-330.

GÓMEZ-MEJÍA, Luis; David BALKIN y Robert CARDY 2001 Dirección y gestión de recursos humanos. $3^{a}$ edición. Madrid: Pearson Educación S.A..

\section{HACKMAN y OLDHAM}

1976 Motivation through the design of work: Test of a theory. Organizational Behavior and Human Performance,16, pp.250-279.

HOOIJBERG Y PETROCK

1993 «On Cultural Change: Using the Competing Values Framework to Help Leaders Execute a Transformational Strategy». En: Human resource Management 32, pp. 29-50. 
IYER, Bala y Thomas DAVENPORT

2008 «Reverse Engineering Google's Innopvation Machine». En: Harvard Business Review, Vol. 86, No 4. Cambridge, MA: Harvard Business Publishing, pp. 59-68.

\section{MILES y SNOW}

1978 Organizational Strategy, Structure and Process. New York, Estados Unidos: Editorial Mc.Graw Hill.

MINTZBERG, Henry

1989 Mintzberg y la dirección. Madrid, España: Ediciones Díaz de Santos S.A.

MUÑOZ-SECA, Beatriz y Joseph RIVEROLA

2000 «Introducción: Clasificación de los casos». En: Casos iberoamericanos de innovación en operaciones. España: Fundación COTEC para la Innovación Tecnológica, pp. 13-21.

MUZYKA, Daniel

2004. "The search for opportunity». En: Financial Times, 17 de setiembre, pp. 2-3. Special Report «Mastering Innovation», primera parte.

PORTER, Michael

1982 Estrategia Competitiva. México:Editorial CECSA.

QUINN

1988 «Beyond Rational Management: Mastering the Paradoxes and competing Demands of High Performance». San Francisco, Estados Unidos: Jossey-Bass.

\section{ROCKS, David y Moon IHLWAN}

2004 «Samsung Design». En: Business Week, diciembre 6, pp. 88-96.

\section{SAWHNEY, Monhabir y Robert WOLCOTT}

2004. «The seven myths of innovation». En: Financial Times, 24 de setiembre, pp. 2-3. Special Report «Mastering Innovation», segunda parte.
SIEMENS

2004a «Generation Three- Contrasting visions for innovation in telecommunications and digital computing». En: The Wall Street Journal Europe, 4 de noviembre, p. A8.

2004b «Innovation- a hybrid of invention and utility». En: The Wall Street Journal Europe, 14 de octubre, p. A5.

STEWART, Thomas y Anand RAMAN

2008 «Finding a Higher Gear». En: Harvard Business Review, Vol. 86, № 7/8. Cambridge, MA: Harvard Business Publishing, pp. 69-76.

SULL, Donald y Alejandro RUELAS-GOSSI

2004 «The art of innovating on a shoestring». En: Financial Times, 24 de setiembre, pp. 1011. Special Report «Mastering Innovation», segunda parte.

\section{TEECE, David}

1986 «Profiting from technological innovation: Implications for integration, collaboration, licesing and public policy». Research Policy 15, pp. 285-306.

TUSHMAN, Michael y Charles III O'RELLY 1998 Innovación. México: Prentice may.

VON HIPPEL; Erick S. THOMKE y Mary SONNACK 1999 «Creating breakthroughs at 3M». En: Harvard Business Review, Vol. 77, № 5. Cambridge, MA: Harvard Business Publishing, setiembreoctubre, pp. 47-57,183.

WATERS, Richard; Simon LONDON y Scott MORRISON

2004 «Hewlett-Packard wants a foot in every market. Can the company do it all?». En: Financial Times, 14 de octubre, p. 13.

WOODMAN, Richard; John SAWYER y Ricky GRIFFIN 1993 «Toward a theory of organizational creativity». En: The Academy of Management Review, Vol. 18, № 2, abril, pp. 293-321. 


\section{ANEXOS}

Anexo 1

Cambio incremental versus Cambio radical

\begin{tabular}{|l|l|}
\hline \multicolumn{1}{|c|}{ Cambio incremental } & \multicolumn{1}{c|}{ Cambio radical } \\
\hline Progreso continuo & Se rompen paradigmas \\
\hline Afecta a parte de la organización & Transforma a la organización entera \\
\hline $\begin{array}{l}\text { Se da por medio de la estructura y los procesos } \\
\text { existentes }\end{array}$ & Se crean nueva estructura y procesos \\
\hline Se producen mejoras tecnológicas & Se dan grandes avances tecnológicos \\
\hline Se mejoran los productos & Se crean nuevos productos y mercados \\
\hline
\end{tabular}

Fuente: DAFT, Richard. Organization Theory and Design. 2004. Octava Edición.Thomson South- Western

Anexo 2

Tipología de la cultura corporativa

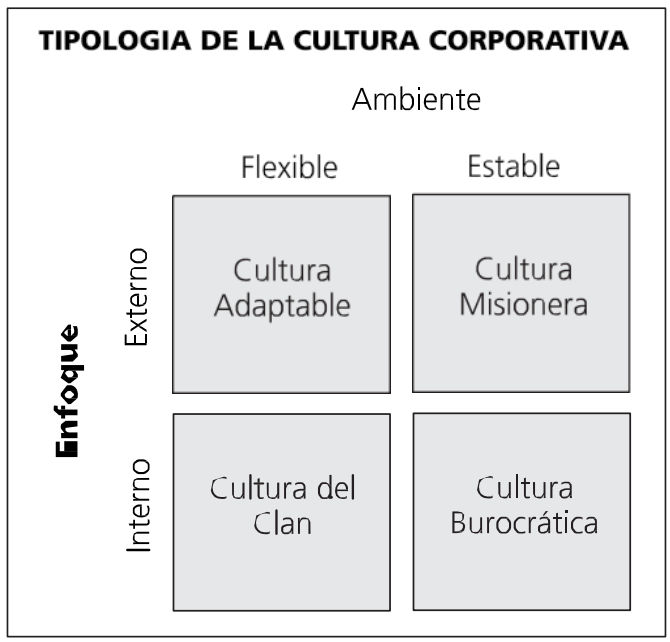

Fuente: DAFT, Richard. Organization Theory and Design. 2004. Octava Edición.Thomson South- Western

\section{Anexo 3}

Modelo de los 5 pilares para la creatividad

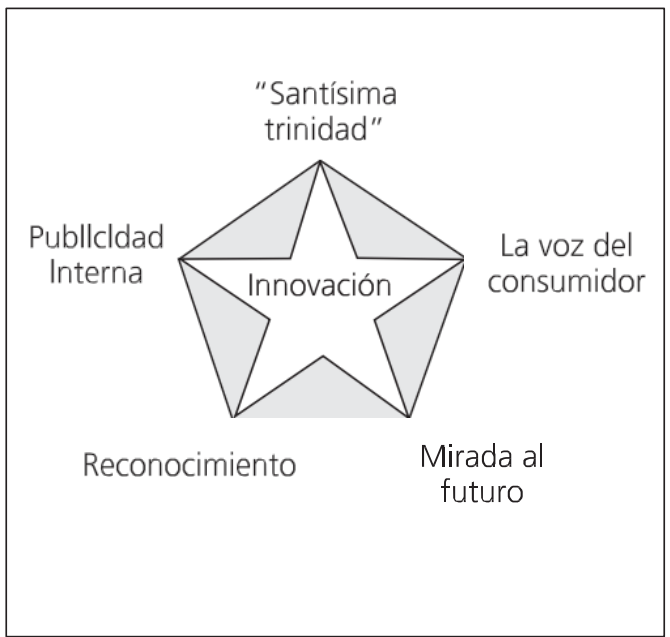

Fuente: Elaboración propia. 